INRA Prod. Anim., 2010, 23 (4), 307-318

\title{
L'apport de lin dans la ration des vaches laitières : Effets sur la production, la composition du lait et des produits laitiers, les émissions de méthane et les performances de reproduction
}

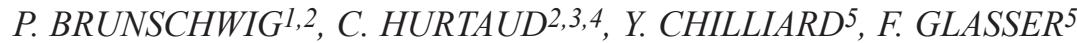 \\ ${ }^{1}$ Institut de l'Elevage, UP Conduite et alimentation du Troupeau Laitier, F-49105 Angers, France \\ 2 INRA, UMT RIEL, UMR Production du Lait, Domaine de la Prise, F-35590 Saint-Gilles, France \\ ${ }^{3}$ INRA, UMR1080, Production du Lait, F-35590 Saint-Gilles, France \\ ${ }^{4}$ Agrocampus Ouest, UMR1080, Production du Lait, F-35000 Rennes, France \\ ${ }^{5}$ INRA, UR1213, Herbivores, F-63122 Saint-Genès Champanelle, France \\ Courriel : Philippe.Brunschwig@inst-elevage.asso.fr
}

La supplémentation en lin des rations des vaches laitières est une pratique qui se développe actuellement, avec un double objectif: l'amélioration de la qualité nutritionnelle du lait (composition en acides gras) et la diminution des émissions de méthane.

L'incorporation de lin dans la ration de vaches laitières est une pratique qui est réapparue depuis une quinzaine d'années. Initialement pratiquée pour diminuer la teneur en matières grasses du lait, l'objectif principal est actuellement de modifier la composition en Acides Gras (AG) des laits à des fins nutritionnelles. De nouvelles applications possibles seraient, en plus, de réduire la production de méthane et d'améliorer la reproduction des vaches. L'équilibre dans le lait entre les grands groupes d'acides gras intéresse le consommateur et la filière laitière. En effet, la réduction de la teneur en acides gras saturés et la diminution du rapport $\mathrm{C} 18: 2 / \mathrm{C} 18: 3$ (acide linoléique/acide linolénique), et éventuellement l'augmentation de la teneur en acide ruménique (CLA cis-9, trans-11) dans le lait, peuvent améliorer sa qualité nutritionnelle pour le consommateur (Shingfield et al 2008). Ceci peut être obtenu en supplémentant les rations avec des oléagineux, en particulier la graine de lin qui est riche en lipides, avec des teneurs élevées en C18:3 n-3 (acide alpha-linolénique), un $\mathrm{AG}$ de la famille des oméga 3. Une filière de lait de consommation et de produits laitiers avec un profil nutritionnel amélioré (Schmitt et al 2006) par l'apport d'une source végétale d'AG oméga 3 (principalement du lin) dans la ration des vaches s'est récem- ment développée, qui démontre l'intérêt de la filière pour ces approches.

L'apport de lin peut être réalisé en élevage sous forme de graines, ayant subi ou non un traitement, ou de tourteaux. Ces matières premières sont introduites directement dans la ration ou bien intégrées dans un aliment concentré venant compléter la ration. Les éleveurs et la filière se posent des questions sur la faisabilité, les conséquences et la pertinence de ces pratiques. Une connaissance scientifique sur ces sujets est disponible : de très nombreux essais et plusieurs synthèses bibliographiques (Palmquist et al 1993, Dewhurst et al 2006, Chilliard et al 2007a et b, Glasser et al 2008) rapportent l'effet d'apport de graines d'oléagineux (dont le lin), de leurs coproduits ou de leur huile sur la teneur en acides gras polyinsaturés du lait.

L'objectif de cet article est de préciser sous quelles formes le lin peut être utilisé, de caractériser les réponses moyennes de l'utilisation de ces différentes formes sur la production du lait et sa composition. L'impact du lin sur la production de méthane sera caractérisé, ainsi que les propriétés du lait et des produits laitiers obtenus avec des rations enrichies en lin. Enfin, l'effet du lin sur des paramètres de reproduction des vaches laitières sera brièvement abordé.

\section{1 / Le lin, un aliment peu cultivé en Europe}

\section{1 / La graine pour un usage} essentiellement non alimentaire

L'Amérique du Nord produit 50\% des graines de lin oléagineux produites dans le monde, suivie par la Chine $(22 \%)$ et l'Inde $(11 \%)$. Le lin oléagineux est peu cultivé dans l'Union Européenne (5\% de la production mondiale), et ne représente qu'une petite surface en France. L'évolution des surfaces en principaux oléagineux (colza, tournesol, soja) est fortement liée au soutien financier associé à la politique de l'UE. Comparativement aux trois autres oléagineux, le lin est resté régulièrement à un niveau très bas de culture, évoluant en dents de scie (tableau 1). Le rendement moyen en lin se situe entre 18 et $20 \mathrm{qx} / \mathrm{ha}$ (de 15 à $25 \mathrm{qx} / \mathrm{ha}$ ). La culture des principaux oléagineux a connu un développement à partir de 2005 avec l'accroissement de leur utilisation non alimentaire (agrocarburants). Parallèlement, le lin a connu une embellie, suite au développement d'une politique contractuelle d'achat de lin oléagineux français pour une destination exclusive vers l'alimentation animale.

L'huile de lin a une très forte propriété siccative, en lien avec l'importante 
Tableau 1. Evolution des oléagineux et du lin non textile en France (d'après Proléa 2008 et Agreste 2008).

\begin{tabular}{|c|c|c|c|c|c|c|c|c|c|c|c|c|c|c|c|}
\hline \multirow{4}{*}{$\begin{array}{l}\text { Colza, tournesol, soja } \\
\text { Surface }(x 1000 \mathrm{ha}) \\
\text { dont jachère et } A C E^{*} \\
\text { Production }(\times 1000 \mathrm{~T})\end{array}$} & 1973 & 1981 & 1986 & 1991 & 1996 & 1999 & 2000 & 2001 & 2002 & 2003 & 2004 & 2005 & 2006 & 2007 & 2008 \\
\hline & 366 & 634 & 1265 & 1872 & 1865 & 2262 & 2014 & 1910 & 1739 & 1863 & 1785 & 1929 & 2095 & 2166 & 2083 \\
\hline & & & & & 248 & 394 & 377 & 331 & 333 & 339 & 298 & 494 & 734 & 998 & 540 \\
\hline & 700 & 1406 & 3063 & 4990 & 5100 & 6640 & 5589 & 4766 & 5066 & 5035 & 5541 & 6161 & 5633 & 6034 & 6420 \\
\hline Lin non textile & 2 & 2 & 1 & 3 & 8 & 19 & 16 & 11 & 8 & 6 & 6 & 12 & 20 & 17 & - \\
\hline dont jachère & - & - & 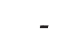 & - & 4 & 1,9 & 1,9 & 1,3 & 1 & 1 & 0,3 & 0,9 & 1,1 & 0,1 & 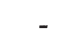 \\
\hline Production $(x 1000 \mathrm{~T})$ & 4 & 4 & 3 & 5 & 15 & 38 & 33 & 23 & 17 & 11 & 13 & 25 & 43 & 32 & 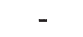 \\
\hline Trituration $(\times 1000 \mathrm{~T})$ & 54 & 24 & 3 & 5 & 3 & - & - & - & - & - & - & - & - & - & - \\
\hline
\end{tabular}

$\left(^{*}\right)$ Aide aux cultures énergétiques.

insaturation des acides gras longs. Les usages industriels consomment plus de 90\% de l'huile de lin produite dans l'UE (encres, peintures, revêtements...; F. Labalette communication personnelle). L'utilisation alimentaire est minoritaire et stagnante. Son autorisation à la commercialisation comme denrée pour l'alimentation humaine est très récente en France (JOF 10 décembre 2008), alors que l'huile de lin était déjà commercialisée pour cet usage dans l'Europe du Nord.

La trituration de graines de lin pour l'extraction d'huile en France ne représentait que $1 \%$ des oléagineux dans les années 80. Elle n'est plus répertoriée après 1996 parce qu'elle ne concerne plus qu'une seule entreprise.

L'UE importe plus des $3 / 4$ de ses besoins en graines de lin et les triture, essentiellement en Belgique et en Allemagne. L'UE consomme tous ses tourteaux de lin et les $2 / 3$ de l'huile de lin extraite. Le fournisseur majoritaire en graines de lin est le Canada $(>80 \%)$.

En France, la consommation apparente d'huile (production + importations - exportations) a connu une baisse régulière jusqu'en 2003. Un regain d'utilisation semble apparaître ensuite. Le niveau d'utilisation des tourteaux de lin se situe autour de $120000 \mathrm{~T}$ (tableau 2). En 2006, les fabricants français d'aliments du bétail ont introduit $64800 \mathrm{~T}$ de tourteau de lin et $27100 \mathrm{~T}$ de graines de lin (oléagineux et textile) dans les concentrés commercialisés (toutes espèces), ce qui représentait respectivement $1,2 \%$ des tourteaux et $3,4 \%$ des graines protéagineuses et oléagineuses utilisés dans ces aliments (Agreste 2006). L'utilisation de tourteaux de lin sous forme de matière première en l'état représenterait $50 \%$ du tonnage utilisé en élevage. Ce type de consommation est connu en finition d'animaux à viande de qualité.

\section{2 / Composition et valeur ali- mentaire des graines, tourteaux et huile de lin}

Ces éléments sont connus en majorité à partir de données provenant d'analyses effectuées sur des lots commerciaux de matières premières entrant dans la fabrication industrielle des aliments pour animaux (Sauvant et al 2004).

On distingue les graines de lin conservées en l'état, qui peuvent être distribuées entières, broyées ou aplaties, et introduites dans la ration collective ou bien dans les aliments concentrés. La teneur en Matières Grasses brutes (MG) est élevée (tableau 3). La teneur en lysine digestible (LysDI) est limitante. Le broyage ou l'aplatissage libèrent peu d'huile, mais rendent la matière première plus rapidement dégradable dans le rumen.

Pour la graine de lin, ce sont les données 2010 (AFZ, io-La Banque de

Tableau 2. Consommation apparente de lin oléagineux en France (production + importations - exportations).

\begin{tabular}{|l|l|l|l|l|l|l|l|l|l|l|l|l|}
\hline & $\mathbf{1 9 7 3}$ & $\mathbf{1 9 8 1}$ & $\mathbf{1 9 8 6}$ & $\mathbf{1 9 9 1}$ & $\mathbf{1 9 9 6}$ & $\mathbf{2 0 0 0}$ & $\mathbf{2 0 0 1}$ & $\mathbf{2 0 0 3}$ & $\mathbf{2 0 0 4}$ & $\mathbf{2 0 0 5}$ & $\mathbf{2 0 0 6}$ & $\mathbf{2 0 0 7}$ \\
\hline Huile (x 1000 T) & 26 & 11 & $\mathbf{8}$ & 10 & 4 & $\mathbf{3}$ & $\mathbf{2}$ & 1 & 5 & 4 & 4 & 6 \\
\hline Tourteaux (x 1000 T) & 114 & 82 & 101 & 128 & 81 & 123 & 127 & 130 & 116 & 120 & 120 & 130 \\
\hline Part du lin dans les tourteaux (\%) & 4,6 & 1,9 & 1,9 & 2,3 & 1,4 & 1,7 & 1,8 & 1,8 & 1,7 & 1,7 & 1,8 & 1,8 \\
\hline
\end{tabular}

Source : Proléa 2008.

Tableau 3. Composition et valeur alimentaire de la graine et du tourteau de lin (par $\mathrm{kg}$ de MS).

\begin{tabular}{|l|l|c|c|c|c|c|c|c|c|c|c|c|c|}
\hline & $\boldsymbol{n}$ & $\begin{array}{c}\text { MS } \\
(\mathbf{\%})\end{array}$ & $\begin{array}{c}\mathbf{M M} \\
\mathbf{( g )}\end{array}$ & $\begin{array}{c}\text { MAT } \\
\mathbf{( g )}\end{array}$ & $\begin{array}{c}\mathbf{C B} \\
\mathbf{( g )}\end{array}$ & $\begin{array}{c}\text { MG } \\
\mathbf{( g )}\end{array}$ & $\mathbf{U F L}$ & $\begin{array}{c}\text { PDIN } \\
(\mathbf{g})\end{array}$ & $\begin{array}{c}\text { PDIE } \\
(\mathbf{g})\end{array}$ & $\begin{array}{c}\mathbf{P}_{\text {abs }} \\
(\mathbf{g})\end{array}$ & $\begin{array}{c}\mathbf{C a}_{\text {abs }} \\
(\mathbf{g})\end{array}$ & $\begin{array}{c}\text { LysDI } \\
(\boldsymbol{\%} \text { PDIE) }\end{array}$ & $\begin{array}{c}\text { MetDI } \\
(\mathbf{\%} \text { PDIE) }\end{array}$ \\
\hline Graine de lin & 75 & $\begin{array}{c}90,3 \\
( \pm 2,3)\end{array}$ & $\begin{array}{c}48 \\
( \pm 10)\end{array}$ & $\begin{array}{c}250 \\
( \pm 28)\end{array}$ & $\begin{array}{c}102 \\
( \pm 29)\end{array}$ & $\begin{array}{c}362^{1} \\
( \pm 35)\end{array}$ & 1,58 & 161 & 84 & 5,0 & 2,3 & 5,9 & 1,9 \\
\hline $\begin{array}{l}\text { Graine de lin } \\
\text { extrudée }\end{array}$ & - & 90,3 & 48 & 250 & 102 & 362 & 1,58 & 189 & 151 & 5,0 & 2,3 & 5,3 & 1,8 \\
\hline $\begin{array}{l}\text { Graine de lin } \\
(2010)^{*}\end{array}$ & 284 & $\begin{array}{c}91,3 \\
( \pm 1,6)\end{array}$ & $\begin{array}{c}43 \\
( \pm 9)\end{array}$ & $\begin{array}{c}239 \\
( \pm 23)\end{array}$ & $\begin{array}{c}103 \\
( \pm 29)\end{array}$ & $\begin{array}{c}406^{2} \\
( \pm 39)\end{array}$ & & & & & & & \\
\hline $\begin{array}{l}\text { Tourteau de } \\
\text { lin «expeller» }\end{array}$ & 292 & $\begin{array}{c}90,4 \\
( \pm 1,8)\end{array}$ & $\begin{array}{c}65 \\
( \pm 8)\end{array}$ & $\begin{array}{c}342 \\
( \pm 27)\end{array}$ & $\begin{array}{c}113 \\
( \pm 17)\end{array}$ & $\begin{array}{c}90 \\
( \pm 24)\end{array}$ & 1,07 & 244 & 190 & 6,1 & 2,6 & 5,6 & 1,8 \\
\hline $\begin{array}{l}\text { Tourteau de } \\
\text { lin déshuilé }\end{array}$ & 39 & $\begin{array}{c}88,6 \\
( \pm 1,8)\end{array}$ & $\begin{array}{c}66 \\
( \pm 8)\end{array}$ & $\begin{array}{c}359 \\
( \pm 25)\end{array}$ & $\begin{array}{c}110 \\
( \pm 15)\end{array}$ & $\begin{array}{c}34 \\
( \pm 12)\end{array}$ & 1,00 & 256 & 202 & 6,1 & 2,8 & 5,6 & 1,8 \\
\hline
\end{tabular}

Source : d'après Sauvant et al 2004 ; *AFZ, io-La Banque de données de l'Alimentation Animale 2010.

1 extraction sans hydrolyse préalable ; 2 avec hydrolyse 
Données de l'Alimentation Animale 2010) qui sont désormais les plus représentatives, en particulier pour la teneur en MG. Les valeurs pour la graine extrudée doivent aussi être réajustées en fonction des données «graines crues» de 2010.

La graine de lin peut être traitée par un processus industriel de «cuisson-extrusion», souvent dénommé extrusion. Ce procédé consiste à appliquer sur des graines préalablement moulues, voire préchauffées dans une atmosphère plus ou moins humide, une forte pression et une température supérieure à $80^{\circ} \mathrm{C}$ pendant un temps très court (moins de 30 secondes) suivies d'un passage forcé dans une filière à l'aide d'une ou plusieurs vis. Ce processus permet la destruction d'une grande partie des composés cyanogènes contenus dans la graine de lin. La teneur en MG n'est pas modifiée, mais l'huile est présente sous forme libre en plus grande proportion que dans la graine entière ou moulue. La dégradabilité théorique de l'azote $\left(\mathrm{DT}_{\mathrm{N}}=50 \%\right)$ est réduite en moyenne d'un tiers. Ce niveau pourra varier selon le niveau de température appliqué et sa durée ; ces caractéristiques du traitement ne sont pas disponibles.

Les tourteaux de lin se distinguent selon le procédé d'extraction. L'utilisation de presse afin d'extraire l'huile à froid pour différents usages, en vue de préserver l'intégrité des insaturations des acides gras présents dans l'huile, produit un tourteau de lin «expeller». La teneur en huile résiduelle dans le tourteau, de l'ordre de $10 \%$, varie selon les caractéristiques de la presse (procédé industriel ou artisanal, puissance, débit). La valeur protéique le rapproche d'un tourteau de colza industriel.

Le procédé consistant en pressage de la graine de lin suivi par une extraction de l'huile résiduelle du tourteau gras par un solvant donne lieu à un tourteau de lin déshuilé, à valeur énergétique un peu moindre. Ce type de tourteau est peu fréquent.

La composition en acides gras de l'huile de lin est présentée au tableau 4. Les acides gras énoncés représentent 97\% des AG Totaux (AGT) présents dans la MG (Sauvant et al 2004). Les acides gras insaturés constituent $90 \%$ des AGT présents. La teneur moyenne en C18:3 n-3 des graines de lots commerciaux observés en France est située en dessous de la valeur seuil des variétés de graines européennes. La composition de l'huile de lin utilisée dans les essais zootechniques (Glasser et al 2008) est très proche de celle des lots commerciaux citée par Sauvant et al (2004).

D'après une campagne d'analyses conduite par l'Onidol en 2005-2006, les variétés «hiver» de lin oléagineux cultivées en France seraient plus riches en acide linolénique de 2 à 4 points comparativement aux variétés de «printemps» (F. Labalette communication personnelle).

Pour un même type, hiver ou printemps, il existe une variabilité de 8 à 10 points entre les variétés les plus pauvres et les variétés les plus riches en C18:3 n-3. Des variétés spéciales peuvent afficher une teneur moyenne de 63 à $65 \%$ de $\mathrm{C} 18: 3$ n-3 selon les années. Actuellement, ce critère n'est pas particulièrement pris en compte par les triturateurs.

Les graines de teillage, issues du lin textile, sont parfois vendues pour l'alimentation animale (si tri préalable des graines). Elles ont des teneurs plus faibles en huile que celles du lin oléagineux ; leur usage dans les aliments pour les enrichir en acides gras insaturés nécessite de connaître leur teneur en C18:3 n-3.

L'origine de l'approvisionnement a de l'influence sur la composition en acides gras du produit final. Des données

Tableau 4. Composition de l'huile de lin en acides gras (en \% des acides gras totaux).

\begin{tabular}{|l|c|c|c|c|}
\hline Nature & France* $^{*}$ & $\begin{array}{c}\text { Moyenne } \\
\text { de 22 essais } \\
\text { zootechniques }^{* *}\end{array}$ & Europe $^{* * *}$ & Canada*** $^{*}$ \\
\hline C14:0 & 0,1 & 0,1 & & \\
\hline C16:0 & 6,4 & 6,1 & $4-6$ & $5-6$ \\
\hline C16:1 n-7 & 0,1 & 0,1 & $<0,5$ & $<0,3$ \\
\hline C18:0 & 3,4 & 3,4 & $2-3$ & $3-4$ \\
\hline C18:1 n-9 & 18,7 & 18,8 & $10-22$ & $19-20$ \\
\hline C18:2 n-6 & 14,7 & 16,3 & $12-18$ & $14-16$ \\
\hline C18:3 n-3 & 54,2 & 54,4 & $56-71$ & $54-61$ \\
\hline C20:0 & & & $<0,5$ & $<0,5$ \\
\hline C20:1 n-9 & & & $<0,6$ & $<0,6$ \\
\hline
\end{tabular}

Sources : Sauvant et al 2004*, Glasser et al $2008^{* *}$, Manuel des corps gras $1992^{\star * *}$

anciennes (1992) montraient que les graines en provenance d'Europe et du Canada avaient une gamme de teneur en acide linolénique plus élevée que celle provenant d'autres régions, telles que l'Argentine ou l'Inde dont les teneurs en C18:3 n-3 étaient respectivement de 45 à $53 \%$ et de 50 à $61 \%$ des AGT.

Les fabricants d'aliments utilisant des graines de lin, dans l'objectif d'un enrichissement en Acides Gras PolyInsaturés (AGPI), recherchent une teneur minimale en C18:3 du lot acheté et pour cela, choisissent les variétés ou s'assurent d'une provenance. Les graines de lin extrudées, actuellement commercialisées dans les aliments composés présentés sous forme de farine ou en granulés, sont associées à des tourteaux et/ou des graines d'oléagineux.

\section{2 / Les effets de l'apport de lin dans la ration sous diffé- rentes formes}

Pour étudier l'effet du lin sur les résultats zootechniques et la composition en AG du lait, nous avons compilé les données bibliographiques disponibles ayant comparé un régime témoin (sans supplément lipidique) avec un ou des régimes supplémentés en lin. Nous avons ainsi collecté les données de 41 essais (liste disponible auprès des auteurs), correspondant à 5 supplémentations en graines écrasées, 14 graines entières, 17 graines extrudées, 5 graines broyées (farine), 4 graines protégées au formol, 5 graines protégées au xylose, 16 huiles, 4 graines micronisées, 1 savon de calcium d'huile de lin et 2 tourteaux. Les quantités de lin apportées par les différentes formes de suppléments ont toutes été ramenées à la quantité de lipides ajoutés dans la ration (dans les quelques cas où la teneur en lipides n'était pas rapportée dans la publication, nous avons utilisé la valeur des tables Inra-AFZ, Sauvant et al 2004).

\section{1 / Les effets des différents types de suppléments}

Les expériences de supplémentation en lipides de lin se distinguent d'abord par le type de supplément apporté : graines sous différentes formes (crues entières, aplaties ou écrasées, moulues en farine, chauffées, extrudées...), tourteaux gras, huile, savons de calcium, protection des graines par traitement au formol, ou de l'huile par encapsulation. Les résultats des régimes témoins, et les effets des différents suppléments (en différence par rapport aux régimes témoins respectifs dans chaque publication) figurent dans le tableau 5. 
Tableau 5. Caractéristiques des régimes témoins et effet de l'ajout des lipides de lin sous différentes formes (en différence par rapport aux témoins, les chiffres en gras indiquent une différence significative).

\begin{tabular}{|c|c|c|c|c|c|c|c|}
\hline & $\begin{array}{l}\text { Régimes } \\
\text { Témoins }\end{array}$ & Graine entière & Graine écrasée & Graine extrudée & Farine & Huile & $\begin{array}{l}\text { Protégé } \\
\text { au formol }\end{array}$ \\
\hline Lipides ajoutés (g/j) & & $650,7 \pm 63,5$ & $1069 \pm 218(4)$ & $362,9 \pm 62,1(17)$ & $557,4 \pm 67,8(5)$ & $516,3 \pm 69,3(18)$ & $413 \pm 120(6)$ \\
\hline$\% \mathrm{MG}$ de la ration & $3,4 \pm 0,22(27)$ & $6,28 \pm 0,38(12)$ & $8,89 \pm 0,72(4)$ & $5,48 \pm 0,65$ & $4,36 \pm 0,42(2)$ & $6,32 \pm 0,76(9)$ & $6,13 \pm 0,8(6)$ \\
\hline MSI (kg/j) & $20 \pm 0,46(39)$ & $-0,51 \pm 0,27(13)$ & $-2,9 \pm 1,65(4)$ & $-0,66 \pm 0,28(13)$ & $-1,14 \pm 0,38(5)$ & $-0,72 \pm 0,49(11)$ & $+0,53 \pm 0,72(3)$ \\
\hline Lait (kg/j) & $27,3 \pm 1,02(44)$ & $-0,07 \pm 0,72(14)$ & $-0,86 \pm 0,89(5)$ & $-0,59 \pm 0,48(15)$ & $-1,65 \pm 0,28(5)$ & $-0,07 \pm 0,6(15)$ & $-0,91 \pm 0,36(3)$ \\
\hline $\mathrm{TB}(\mathrm{g} / \mathrm{kg})$ & $39 \pm 0,8(44)$ & $+0,2 \pm 0,7(14)$ & $+1,3 \pm 1,6(5)$ & $-3,2 \pm 0,8(15)$ & $+0,8 \pm 0,7(5)$ & $-2,0 \pm 1,2(15)$ & $+2,0 \pm 1,6(3)$ \\
\hline $\mathrm{TP}(\mathrm{g} / \mathrm{kg})$ & $33 \pm 0,4(41)$ & $-0,5 \pm 0,4(14)$ & $+1,3 \pm 1,4(5)$ & $-0,5 \pm 0,2(15)$ & $-0,8 \pm 0,4(4)$ & $+0,0 \pm 0,3(13)$ & $+0,4 \pm 0,2(3)$ \\
\hline $\mathrm{TL}(\mathrm{g} / \mathrm{kg})$ & $48 \pm 0,4(24)$ & $-0,2 \pm 0,2(10)$ & $+0,6 \pm 0,4(2)$ & $+0,3 \pm 0,5(4)$ & $+0,7 \pm 0,1(3)$ & $+0,6 \pm 0,3(12)$ & - \\
\hline \multicolumn{8}{|c|}{ AG du lait (\% AG totaux) } \\
\hline C4-C14 & $25,6 \pm 0,87(32)$ & $-4,38 \pm 0,62(11)$ & $-5,62 \pm 1,01(4)$ & $-4,71 \pm 1,24(9)$ & $-5,26 \pm 1,58(4)$ & $-6,04 \pm 1,38(12)$ & $-2,45 \pm 0,33(3)$ \\
\hline C16:0 & $31,1 \pm 0,72(40)$ & $-6,27 \pm 0,83(14)$ & $-9,23 \pm 1,41(5)$ & $-7,88 \pm 1,74(9)$ & $-7,75 \pm 1,53(5)$ & $-7,68 \pm 1,33(14)$ & $-3,93 \pm 0,56(3)$ \\
\hline C18:0 & $9,9 \pm 0,43(40)$ & $+4,31 \pm 0,39(14)$ & $+3,72 \pm 0,84(5)$ & $+3,21 \pm 0,85(9)$ & $+5,5 \pm 0,88$ & $+2,59 \pm 0,6(14)$ & $+1,6 \pm 0,17(3)$ \\
\hline 18:1-oléique & $19,9 \pm 0,6(40)$ & $+5,75 \pm 0,7(14)$ & $+8,26 \pm 1,41(5)$ & $+4,36 \pm 1,19(9)$ & $+5,4 \pm 1,05(5)$ & $+3,32 \pm 0,79(14)$ & $+1,04 \pm 0,8(3)$ \\
\hline 18:1-trans & $4 \pm 0,45(37)$ & $+1 \pm 0,49(11)$ & $+1,88 \pm 1,15(5)$ & $+4,07 \pm 1,22(9)$ & $+1,33 \pm 0,79(4)$ & $+4,8 \pm 0,84(14)$ & $+0,84 \pm 0,75$ \\
\hline $18: 1-t 10$ & $0,5 \pm 0,11(16)$ & $+0,02 \pm 0,16(2)$ & - & $+0,83 \pm 0,32(7)$ & - & $+0,66 \pm 0,25(12)$ & - \\
\hline $18: 1-t 11$ & $1,6 \pm 0,15(31)$ & $+0,49 \pm 0,24$ & $+0,8 \pm 0,61(5)$ & $+0,9 \pm 0,44(8)$ & - & $+1,72 \pm 0,51(14)$ & - \\
\hline $18: 1-t 9$ & $0,3 \pm 0,05$ (19) & $+0,15 \pm 0,09(4)$ & - & $+0,14 \pm 0,04(7)$ & $+0,11 \pm 0,04(2)$ & $+0,2 \pm 0,03(12)$ & - \\
\hline $18: 2 n-6$ & $2,2 \pm 0,11(40)$ & $+0,01 \pm 0,13(14)$ & $+0,24 \pm 0,27(5)$ & $+0,09 \pm 0,14(9)$ & $-0,07 \pm 0,25(5)$ & $-0,11 \pm 0,05(14)$ & $+1,36 \pm 0,36(3)$ \\
\hline CLA-c9t11 & $0,7 \pm 0,07(25)$ & $+0,1 \pm 0,09(6)$ & - & $+0,34 \pm 0,14(8)$ & $+0,04 \pm 0,17(3)$ & $+0,75 \pm 0,22(13)$ & - \\
\hline $18: 3 n-3$ & $0,5 \pm 0,04(39)$ & $+0,4 \pm 0,05(14)$ & $+0,87 \pm 0,18(5)$ & $+0,52 \pm 0,08(9)$ & $+0,75 \pm 0,1(5)$ & $+0,24 \pm 0,07(14)$ & $+3,08 \pm 1,38(3)$ \\
\hline AG Saturés & $67,2 \pm 0,93(37)$ & $-6,29 \pm 0,8(14)$ & $-10,38 \pm 1,82(4)$ & $-9,41 \pm 2,05(9)$ & $-7,71 \pm 2,26(4)$ & $-11,19 \pm 2,16(14)$ & $-4,38 \pm 0,79(3)$ \\
\hline AGMI & $26,5 \pm 0,83(40)$ & $+6 \pm 0,72(14)$ & $+9,69 \pm 2,22(5)$ & $+8,37 \pm 1,98(9)$ & $+6,11 \pm 1,36(5)$ & $+8,17 \pm 1,63(14)$ & $+0,52 \pm 1,57(3)$ \\
\hline AGPI & $3,5 \pm 0,14(40)$ & $+0,48 \pm 0,19(14)$ & $+1,33 \pm 0,33(5)$ & $+1,45 \pm 0,33$ (9) & $+1,09 \pm 0,4(5)$ & $+2,05 \pm 0,56(14)$ & $+4,41 \pm 1,75(3)$ \\
\hline $18: 2 / 18: 3$ & $4,8 \pm 0,36(40)$ & $-2,03 \pm 0,44(14)$ & $-3,41 \pm 0,39(5)$ & $-2,58 \pm 0,71(9)$ & $-2,21 \pm 0,7(5)$ & $-1,37 \pm 0,34(14)$ & $-4,32 \pm 0,22(3)$ \\
\hline
\end{tabular}

Les chiffres sont des moyennes \pm erreur type, avec le nombre de données disponibles entre parenthèses.

Les quantités moyennes apportées dans ces essais sont importantes, de 362 à $650 \mathrm{~g} / \mathrm{j}$ selon les suppléments, et jusqu'à $1069 \mathrm{~g} / \mathrm{j}$ pour les graines écrasées. Les rations supplémentées étaient donc en moyenne à 5-6\% de matières grasses.

a) Sur les performances zootechniques

Globalement, la quantité de Matière Sèche Ingérée (MSI) est légèrement diminuée par les différents suppléments (non significativement, sauf pour la graine extrudée et la farine). L'apport de graines protégées se traduit par une augmentation significative. Une revue récente (Petit 2010) conclut également à l'absence d'effet significatif de la supplémentation en graines de lin sur les quantités ingérées. Les quantités de lait produites ne sont pas significativement diminuées, sauf pour la farine. Les taux butyreux et protéique restent en moyenne inchangés sur ces données, sauf pour la graine extrudée, qui provoque une baisse moyenne de 3,2 points de TB et 0,5 point de TP. La chute moyenne de 2 points observée avec l'huile n'est pas significative du fait d'une grande variabilité des réponses. Les comptages de cellules somatiques du lait ne semblent pas non plus affectés (Petit 2010).

\section{b) Sur la composition en $A G$ du lait}

Les variations de la composition en $\mathrm{AG}$ du lait sont conformes à ce que l'on sait de l'effet des matières grasses végétales: une baisse des AG courts et moyens (du C4 au C16) et une augmentation des AG longs en C18, en particulier des polyinsaturés (AGPI).

Quand on compare les effets des différents types de suppléments, on observe que les graines protégées affectent moins le profil global des AG (moins d'effet sur les AG courts et moyens, moins d'effet sur les C18:1-trans), mais augmentent davantage les pourcentages d'AGPI, à la fois C18:2 n-6 et C18:3 n-3. Cela est dû à la protection de ces lipides contre la biohydrogénation par les microbes du rumen, et donc à une moindre production d'AG trans (dont certains inhibent la synthèse de matières grasses par la mamelle) à partir des AGPI de la ration. Quand les lipides sont protégés de cette biohydrogénation, les AG trans sont donc moins élevés, et les AGPI protégés se retrouvent davantage dans les produits (ici le lait).

En ce qui concerne les autres suppléments, les principales différences sont les suivantes :
- les C18:1-trans: les huiles et les graines extrudées ont un effet majeur par rapport aux autres suppléments (aux doses pratiquées dans les essais, de 360 à $520 \mathrm{~g}$ de lipides $/ \mathrm{j}$, ils doublent environ la teneur basale (4\%) de ces AG). L'effet sur le CLA-c9t11 est similaire, puisqu'il est très majoritairement synthétisé dans la mamelle à partir du C18:1-t11. La graine extrudée et l'huile augmentent significativement le C18:1$t 10$, qui est corrélé avec la baisse de la synthèse de matières grasses ; cela est cohérent avec les effets inhibiteurs plus importants de ces deux suppléments sur le TB, comparés à ceux de la graine entière ;

- l'acide linoléique (C18:2 n-6) : seules les huiles provoquent sa diminution, les autres suppléments n'ont pas d'effet significatif en moyenne ;

- le C18:3 n-3 : la graine écrasée et la farine sont les formes qui l'augmentent le plus $(+0,87$ et $+0,75$, à partir d'une teneur initiale de $0,5 \%$ des AG). L'huile est la forme qui l'augmente le moins $(+0,24)$. Les graines crues et extrudées sont intermédiaires. Tous suppléments confondus, la teneur maximale en C18:3 n-3 observée est de $2 \%$ des AG du lait (à l'exception de 2 données très atypiques obtenues avec du lin 
formolé). Les teneurs maximales du lait en C18:3 n-3 rapportées dans les publications sont de $1,2 \%$ des $A G$ avec des graines entières, $1,4 \%$ avec des graines extrudées et $1,7 \%$ avec de l'huile (sur une ration riche en concentrés).

Les données sur les autres suppléments sont plus rares, donc nous n'avons pas fait d'analyses statistiques sur ces données. En s'intéressant à l'augmentation du C18:3 n-3, qui reflète le degré de protection des lipides, on peut dire que les savons de calcium ont un effet similaire aux huiles. Cela est cohérent avec le fait que les savons de calcium d'AG insaturés sont dissociés aux $\mathrm{pH}$ du rumen, et libèrent donc les $\mathrm{AG}$, comme dans le cas des huiles. Les tourteaux et les graines traitées au xylose ont un effet protecteur (C18:3 n-3 entre 1,4 et $2 \%$ des AG du lait), mais qui reste inférieur à la protection au formol. Les teneurs maximales en C18:3 n-3 sont limitées par la biohydrogénation de cet AG dans le rumen, et non par une limite d'incorporation dans le lait, puisque des teneurs dépassant $13 \%$ des AG du lait ont été obtenues par une infusion d'huile de lin dans la caillette des vaches (Petit et al 2002).

Les résultats ci-dessus s'appuient majoritairement sur des comparaisons entre publications différentes, compilées dans la base de données. Cependant, un certain nombre de publications ont comparé différents types de suppléments dans la même expérience. Il est toutefois difficile d'en tirer des conclusions, puisque les suppléments ne sont souvent pas comparables entre publications. Les comparaisons reposent donc souvent sur une seule publication, voire vont en sens inverse entre deux publications, et demandent donc d'autres investigations pour pouvoir être interprétées. Les graines extrudées, comparées aux graines crues, augmentent davantage les C18:1-trans et diminuent davantage les AG courts et moyens dans les 3 publications avec ce type de comparaison (Gonthier et al 2005, Akraim et al 2007, Chilliard et al 2009). Plusieurs publications n'observent aucune différence entre types de supplément, sur le TB et le C18:3 n-3 du lait, en comparant des graines crues à des suppléments micronisés, ou extrudés, ou des savons (Mustafa et al 2003, Soita et al 2003, Akraim et al 2007, Cortes et al 2010).

\section{c) Sur les émissions de méthane}

La réduction des émissions de méthane (CH4) par les ruminants est un enjeu important pour limiter les rejets agricoles de gaz à effet de serre. Différents types de suppléments lipidiques sont connus pour réduire les émissions de
$\mathrm{CH} 4$, dont la supplémentation en graine ou en huile de lin. Ainsi, des réductions de 12,38 et $64 \%$ ont été observées en ajoutant à une ration à base d'ensilage de maïs $5 \%$ de lipides du lin sous forme de graines entières, de graines extrudées ou d'huile libre, respectivement (Martin et al 2008). Avec de la graine de lin extrudée, des réductions croissantes de la production de $\mathrm{CH} 4(-10,-16$ et - 41\%) ont été observées lors d'ajouts croissants $(5,10$ et $15 \%$ de graines extrudées dans la ration, respectivement) (Martin et al 2009). Enfin, cet effet semble se maintenir après 18 mois de supplémentation continue en graine de lin extrudée (Martin et al 2010).

En raison de variations simultanées de la méthanogenèse ruminale, de la biohydrogénation ruminale et de la sécrétion des différents $A G$ du lait, il a été possible d'établir des équations de prédiction de la production de $\mathrm{CH} 4$ en fonction de la consommation de fourrage, et du profil des AG du lait (notamment saturés à chaîne moyenne de $\mathrm{C} 8$ à $\mathrm{C} 16$, et monoinsaturés de structure trans), au sein de lots de vaches supplémentées ou non en graine ou huile de lin (Chilliard et al 2009).

\section{2 / Les effets d'autres facteurs, alimentaires et animaux, sur la réponse au lin}

Les données moyennes du tableau 5 donnent des informations générales sur les effets de différents suppléments, mais ces effets sont à nuancer en fonction de plusieurs facteurs. Nous avons étudié 4 facteurs sur une sélection des publications de la base de données, avec uniquement des graines crues, des graines extrudées et de l'huile.

\section{a) La dose apportée}

Le premier facteur est la dose de lipides apportée. Dans une première approche, nous avons compilé les données de différentes publications utilisant des doses diverses de lin. Les C18:1-trans et le CLA-c9t11 augmentent linéairement avec la dose ajoutée pour l'huile et les graines extrudées, mais il n'y a pas d'effet dose pour les graines entières. Le C18:2 n-6 et le C18:3 n-3 ne dépendent pas de la dose apportée, quel que soit le supplément : ils plafonnent pour les fortes doses. Le TB diminue en fonction de la dose ajoutée pour les graines extrudées, mais pas pour les graines entières et l'huile. Les fortes chutes de TB observées par certains auteurs (Loor et al 2005, Flachowsky et al 2006, Chilliard et al 2009, Hurtaud et al 2010) sont corrélées à de fortes teneurs du lait en C18:1-t10. Pour compléter cette première approche, nous nous sommes intéressés aux publications ayant pour objectif spécifique d'étudier l'effet «dose», et dont le protocole comprenait des doses croissantes de lin. Dans les 6 publications que nous avons répertoriées comparant plusieurs doses, seuls Deaville et al (2004) montrent des effets non linéaires sur les AG trans (croissants aux fortes doses) et Flowers et al (2008) sur le C18:3 n-3 (qui plafonne aux fortes doses). Toutes les autres publications mettent en évidence des effets linéaires de la dose de lin, à la fois sur les réponses zootechniques et sur la composition en AG du lait.

\section{b) Le type de fourrage de la ration}

Le deuxième effet testé est celui du fourrage de la ration de base. Cet effet est difficile à distinguer quand on prend déjà en compte l'effet du type et de la dose de supplément. Toutefois, il apparaît que les chutes de TB sont plus prononcées sur les rations à base d'ensilage de maïs. Le C18:1-c9 (oléique) n'est pas significativement augmenté quand on supplémente au pâturage, alors qu'il l'est dans le cas des autres rations. Les AG Saturés (AGS), et les AG courts et moyens $\mathrm{C} 4$ à $\mathrm{C} 14$, sont davantage diminués par les apports d'huile (comparés aux graines crues et extrudées) et sur les rations à base d'ensilage de maïs (comparées au pâturage et ensilage d'herbe). L'effet observé pour les AG MonoInsaturés (AGMI) est la réciproque (davantage augmentés par les huiles et sur ensilage de maïs). Aucun autre effet majeur du fourrage de base n'a été détecté sur notre jeu de données. Les résultats préliminaires d'une étude récente montrent en outre que le rapport trans 10/trans 11 des isomères du C18:1 augmente plus fortement lorsque le lin est ajouté à un régime ensilage de maïs qu'à un régime foin (Chilliard et al 2008).

c) Le pourcentage de concentré de la ration

Le pourcentage de concentrés de la ration est également un facteur qui peut affecter la digestion ruminale des lipides, et donc la composition en AG du lait des vaches supplémentées. Selon deux publications comparant l'effet d'huile de lin sur des rations avec beaucoup (65-70\% de la ration) ou peu (30$35 \%$ ) de concentrés, la majorité des réponses sont similaires avec les deux types de rations. Loor et al (2005) ont montré que le C18:0 et le C18:1-c9 augmentent plus avec une ration pauvre en concentré, que le $\mathrm{C} 16: 0$ diminue plus et que le C18:1-trans 10 et le C18:3 n-3 ont tendance à augmenter moins, et que la sécrétion de matières grasses augmente avec la ration pauvre en concentré alors qu'elle diminue avec la ration riche en concentré. Flachowsky et al 
(2006) confirment que les C18:1-trans ont tendance à augmenter davantage sur une ration riche en concentré. Sur toutes les autres réponses des animaux (TB, autres AG...), ces deux publications ne mettent en évidence que peu de différences significatives dans la réponse à l'huile de lin entre une ration riche ou pauvre en concentrés. Ces données sont très fragmentaires, et on sait par ailleurs (Chilliard et al 2007a) qu'une forte teneur en concentrés de la ration a tendance à favoriser la production d'AG trans au niveau du rumen et donc des produits, et à diminuer le $\mathrm{TB}$, mais cela ne peut pas être démontré à partir des quelques données publiées sur le lin.

\section{d) Stade de lactation}

Les données sur le stade de lactation étaient assez déséquilibrées, avec 6 données en début de lactation (début de l'essai avant $30 \mathrm{j}$ de lactation), 39 en milieu de lactation (début de l'essai entre 30 et $150 \mathrm{j}$ de lactation) et 13 en fin de lactation (début de l'essai à plus de $150 \mathrm{j}$ de lactation). Nous n'avons trouvé aucun effet significatif du stade de lactation sur la production, la composition du lait et le profil en acides gras.

\section{3 / Les propriétés rhéolo- giques et sensorielles du lait et des produits laitiers (beurre et fromages) de vache et de brebis}

\section{1 / Le lait}

L'apport de lin extrudé dans la ration des vaches laitières modifie la structure physique de la matière grasse. Hurtaud et al (2010) ont montré que l'apport de doses croissantes de lin extrudé $(0$, $2,1 \%$ et $4,2 \%$ de la MS de la ration) provoquait une diminution du diamètre des globules gras de la crème.

L'apport d'un concentré contenant des graines de lin extrudées $(1,2 \mathrm{~kg} / \mathrm{j})$ en complément d'un fourrage à base d'ensilage d'herbe $(45 \%)$ et d'ensilage de maïs $(55 \%)$ modifie également le comportement thermique de la matière grasse des laits. Cette matière grasse a une teneur plus faible en matière grasse solide (figure 1, Smet et al 2010) et contient également plus de triglycérides à bas point de fusion et moins de triglycérides à moyen et haut point de fusion (Smet et al 2010).

Les laits issus de vaches supplémentées en graines de lin extrudées $(1,2 \mathrm{~kg} / \mathrm{j})$ réagissent également différemment au traitement thermique de type UHT. La formation de produits primaires d'oxy- dation augmente plus rapidement avec une valeur limite atteinte au bout de 40 jours de stockage en brique. Ces laits semblent donc plus sensibles à l'oxydation (Smet et al 2009).

\section{2 / Le beurre}

$\mathrm{Au}$ niveau nutritionnel, la composition en acides gras du beurre est similaire à celle du lait (Hurtaud et al 2010).

Lors de la transformation beurrière de la crème issue de régimes enrichis en lin extrudé, les pertes de matière grasse dans le babeurre et l'humidité des beurres augmentent linéairement avec la dose de lin extrudé. Avec 4,2\% de lin extrudé dans la ration, les pertes de $\mathrm{MG}$ dans le babeurre ont été deux fois plus importantes qu'avec les autres traitements et les beurres ont été plus humides. Ces résultats peuvent sans doute être reliés à l'effet marqué de cette dose de lin sur le diamètre des globules gras de la crème $(-0,7 \mu \mathrm{m})$. Ces globules gras plus petits ont pu s'échapper dans le babeurre au lieu d'être intégrés au réseau formé dans le beurre. D'après Goudédranche et al (2000) et Michalski (2004), les beurres issus de crèmes ayant des globules gras de petite taille et/ou riches en acides gras insaturés sont plus humides. Cette augmentation de l'humidité serait une conséquence de l'augmentation de la surface membranaire hydrophile associée aux petits globules gras, et donc d'une rétention d'eau accrue (Michalski 2004).
La dureté des beurres, mesurée instrumentalement ou en bouche, a diminué linéairement avec l'accroissement de la dose de lin extrudé et la tartinabilité a été améliorée. L'augmentation des acides gras insaturés (en particulier du C18:1-c9) et la diminution des acides gras saturés (en particulier du C16:0) consécutives à l'augmentation de la dose de lin extrudé dans la ration, qui conduisent à une diminution de la quantité de matière grasse cristallisée, expliqueraient cet effet du lin sur la texture du beurre.

L'accroissement de la dose de lin extrudé dans la ration a entraîné une diminution faible et linéaire de la luminosité et l'indice de jaune des beurres. Ces beurres ont été globalement peu typés au niveau sensoriel. Seule l'odeur de rance a eu tendance à augmenter, mais la note est restée très faible. Cette absence de défauts de goût et de flaveur pourrait paraître surprenante compte tenu de la teneur importante des beurres en acides gras insaturés (Hurtaud et al 2010).

\section{3 / Les fromages}

Les données concernant les vaches laitières sont peu nombreuses. Les résultats sur la fabrication de fromages ont été étudiés sur la vache et la brebis.

Quelle que soit l'espèce (brebis ou vache laitière), la composition en acides gras des fromages est similaire à celle

Figure 1. Teneur en matière solide (\%) de la matière grasse laitière des vaches alimentées avec le régime témoin $(\bullet)$ ou un régime contenant des graines de lin extrudées (o) (Smet et al 2010).

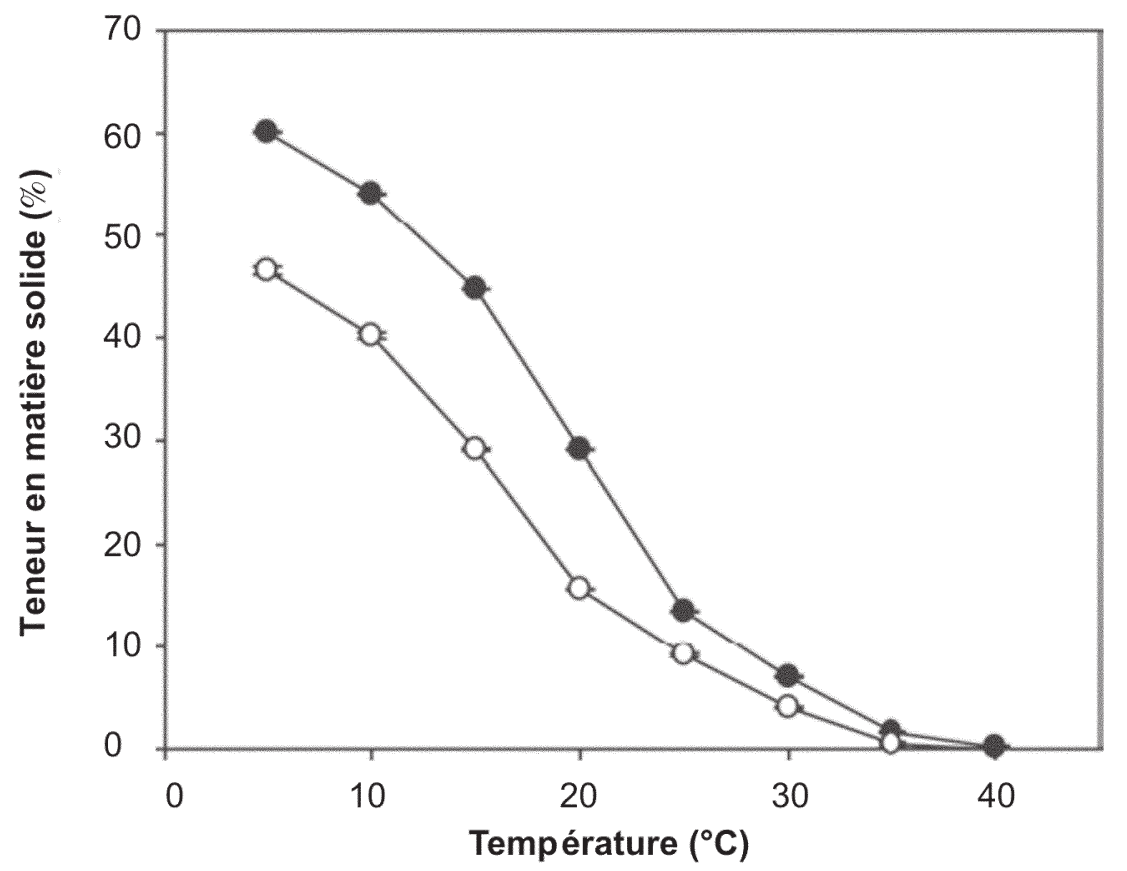


du lait. Selon Gòmez-Cortéz et al (2009) et Luna et al $(2005,2008)$, pour des fromages de type Manchego, le profil en acides gras est similaire à celui du lait. Selon Zhang et al (2006a et b) et Luna et al (2005), sur un autre type de fromages, le procédé de transformation du lait en fromage et l'affinage quelle que soit sa durée, ne modifient pas la composition en acides gras.

\section{a) Fromages de lait de vache}

Des fromages de type Saint-Nectaire ont été fabriqués à partir du lait de vaches ayant reçu $2,4 \mathrm{~kg} / \mathrm{j}$ de lin extrudé dans une ration à base d'ensilage de maïs principalement (Martin et al 2009). Leur texture était moins ferme, plus fondante et plus grasse. Les fromages avaient une croûte plus sombre, leur pâte avait un aspect plus brillant et plus coloré. La modification de ces 2 paramètres serait liée à une exsudation des acides gras polyinsaturés lors des premières étapes de l'affinage.

Des fromages de type Emmental fabriqués avec le lait de vaches ayant reçu $1 \mathrm{~kg} / \mathrm{j}$ de graines de lin présentaient une belle ouverture, mais leur pâte était jugée trop ferme et leur degré de maturité jugé moins avancé (Stoll et al 2003).

La forme d'apport du lin peut avoir un impact sur les propriétés des fromages. De l'Emmental ou du Tilsit ont été fabriqués à partir de laits de vaches ayant reçu $500 \mathrm{~g} / \mathrm{j}$ de matière grasse sous forme de graines de lin crues ou extrudées. Quel que soit le type de fromage, le lin extrudé a entraîné une augmentation de la longueur de pâte, une diminution de la fermeté et la pâte a été mieux notée par un panel de dégustation par rapport au lin cru (Schori et al 2006).

Que ce soit pour les Saint-Nectaire ou les Emmentals, aucun défaut de goût lié à l'oxydation des acides gras n'a été décelé (Stoll et al 2003, Martin et al 2009). Cependant, les Saint-Nectaire avaient une odeur de lait et un arôme plus faibles, une plus forte odeur de foin et un goût aigre moins prononcé (Martin et al 2009).

\section{b) Fromages de lait de brebis}

L'apport de graines de lin broyées $(200 \mathrm{~g} / \mathrm{j})$ dans la ration des brebis permet une amélioration du rendement fromager essentiellement liée à une élévation du taux protéique des laits (Zhang et al 2006a). Le rendement fromager en frais, ou corrigé (37\% d'humidité) a été plus élevé avec le lait des brebis ayant reçu un concentré contenant 9,18 et $26 \%$ de graines de lin pendant 5 semai- nes. Ceci est lié à des taux de solides supérieurs. Les teneurs en MG, protéines et MS ont été similaires entre les fromages. La perte de MG dans le lactosérum a été plus élevée pour le traitement $19 \%$, intermédiaire pour le $26 \%$ et plus faible pour le $6 \%$ et le témoin. (Zhang et al 2006b).

Sur des fromages de type Manchego, Gòmez-Cortéz et al (2009) qui ont apporté 6 et $12 \%$ de graines de lin extrudées à des brebis et Luna et al (2008) avec un mélange de graines de lin et d'huile de tournesol n'ont mis en évidence aucun effet particulier du lin. Tous les fromages ont été évalués positivement pour l'apparence, l'arôme, le goût et la texture et aucune différence n'a été mise en évidence par rapport au témoin. Sur un autre type de fromage, avec un régime enrichi en graine de lin entière (17\% de la MSI), il n'y a eu aucune différence sur l'apparence, le goût et l'acceptabilité des fromages. Bien que les notes sur l'arôme et sur la texture soient plus faibles pour les fromages «lin» après 2 mois d'affinage, les différences n'étaient pas significatives. De plus, à 3 mois d'affinage, les valeurs étaient similaires. Finalement aucune étrange odeur ou flaveur n'a été détectée dans cette étude (Luna et al 2005).

\section{4 / Effets du lin sur la repro- duction}

En Amérique du Nord, les rations des vaches laitières sont couramment supplémentées en matière grasse ou en huile afin d'améliorer le bilan énergétique post-partum. Il a toutefois été montré que cette amélioration n'est généralement pas obtenue, du fait d'une tendance à diminuer l'ingestion accompagnée d'une augmentation de la production laitière et parfois de la sécrétion de matières grasses (Chilliard 1993). Les matières grasses alimentaires pourraient toutefois avoir un effet sur la reproduction en modulant le rôle des AG non estérifiés (AGNE) qui agissent comme un «signal» entre statut nutritionnel et reproduction (Friggens et al 2010). Un effet direct délétère des AGNE sur la viabilité des cellules folliculaires a été démontré in vitro (Vanholder et al 2005, 2006). Une altération de la capacité de synthèse de la progestérone par le corps jaune chez les animaux en déficit énergétique est supposée (Leroy et al 2008).

La supplémentation en acides gras de type oméga 3 pourrait contribuer également à améliorer la reproduction grâce à une meilleure immunité. L'apport de graines de lin entières a provoqué une diminution de la prolifération des cellu- les mononucléaires initiées lors de l'implantation des embryons chez la vache laitière (Lessard et al 2003), ce qui permet une diminution de la mortalité embryonnaire (Petit et Twagiramungu 2006).

Les coques de la graine de lin sont riches en lignanes qui sont des antioxydants forts. Elles augmentent le niveau de réponse endogène en surexprimant des gènes codant pour des enzymes telles que la superoxyde dismutase, la catalase et la peroxydase. Les coques de graines de lin augmentent, dans la glande mammaire, l'activité de la superoxyde dismutase, enzyme responsable de la suppression des radicaux libres conduisant au stress oxydatif. Cela contribuerait à améliorer la reproduction (Petit et al 2009). Toutefois, la supplémentation en graine de lin extrudée a eu tendance à augmenter certains indicateurs plasmatiques de lipoperoxydabilité chez des vaches laitières, effet qui pourrait être inversé par l'emploi d'antioxydants d'origine végétale (Gobert et al 2009, Ferlay et al 2010).

L'apport de graines entières de lin augmente la concentration plasmatique en acides gras oméga 3 (Lessard et al 2003). Zachut et al (2010) en apportant $1 \mathrm{~kg} / \mathrm{j}$ de lipides encapsulés contenant $40,8 \%$ d'huile de lin $(242,2 \mathrm{~g} / \mathrm{j}$ de C18:3 n-3) ont modifié le rapport n-6/n3 dans le plasma qui est passé de 42 avec le témoin à 6,74 avec le lin. Comparativement à un apport de sels de calcium d'huile de palme ou de soja micronisé, le lin a diminué dans un essai les concentrations plasmatiques en AGNE et en $\beta$-hydroxybutyrate et les concentrations hépatiques de lipides et de triglycérides tout en augmentant les concentrations hépatiques de glycogène (Petit et Benschaar 2007). La concentration en progestérone dans le sérum a été significativement plus importante chez les vaches recevant des graines de lin, en particulier du jour 17 au jour $21 \mathrm{du}$ cycle ovarien. Petit et Twagiramungu (2006) ont constaté que les concentrations au pic de progestérone ont tendance à être plus élevées pour les vaches qui ont reçu des graines de lin. Une amélioration de la fertilité pourrait donc être observée (Morris et Diskin 2008).

Le lin aplati a augmenté le diamètre moyen des follicules ovariens (Ambrose et al 2006). Une fertilité plus importante a été rapportée chez des vaches avec de grands follicules sans une augmentation des concentrations en progestérone dans la phase lutéale suivante. L'ovulation de plus grands follicules chez les vaches «lin» pourrait avoir contribué à l'amélioration du taux de conception, peut-être due à un ovocyte plus viable, le nombre de follicules 
et la taille du corps jaune n'ayant pas été affectés. Au contraire, Petit et al (2002) et Petit et Twagiramungu (2006) ont constaté des corps jaunes plus gros avec des régimes très riches en oméga 3 (lin tanné ou mélange lin tanné-huile de poisson) avec une dynamique folliculaire identique. Le nombre moyen de follicules de 2 à $5 \mathrm{~mm}$ aux jours 5 et $9 \mathrm{du}$ cycle a été plus élevé chez des vaches qui recevaient $1 \mathrm{~kg} / \mathrm{j}$ de lipides encapsulés riches en C18:3 (Zachut et al 2010).

Les taux de gestation présumée après la première IA étaient plus importants chez les vaches recevant du lin aplati (Ambrose et al 2006). D'après Petit et al (2002), l'amélioration du taux de gestation observé lors de l'apport d'acide $\alpha$-linolénique pourrait résulter de plus faibles niveaux de production en prostaglandine PGF2 $\alpha$. La mortalité embryonnaire est également plus faible pour les vaches qui ont reçu du lin (Petit et Twagiramungu 2006). L'apport d'une source d'oméga 3 améliorerait la survie embryonnaire dans les troupeaux avec une faible fertilité.

Finalement, l'apport de lin pourrait améliorer le taux de gestation via différents effets potentiels, dont l'impact zootechnique sur les performances de reproduction n'est actuellement pas quantifiable précisément.

\section{5 / Discussion - Conclusion}

Parmi les différentes formes sous lesquelles se présente le lin, les graines et les tourteaux gras apparaissent celles les plus facilement utilisables en élevage bovin laitier.

$\mathrm{Au}$ vu de la variabilité de teneur en AGPI de l'huile ou des graines observée sur les variétés, il serait important de connaître la teneur en C18:3 de la source de lin utilisée. La caractérisation de lots de graines de lin, parfois pratiquée en vue de leur usage dans l'alimentation pour animaux, devrait être étendue aux variétés de lin, oléagineux et textile. Leur usage serait ainsi orienté par le profil en acides gras de leur huile.

L'apport de lipides de lin modifie plusieurs paramètres chez les vaches laitières. On observe généralement une légère chute des quantités ingérées, qui ne se traduit pas par une chute de la production de lait. La réponse en lait est variable et n'est pas aisément prédictible à partir des données publiées. La réponse du TB est également variable, et semble liée à la production de certains isomères trans des AG dans le rumen, plus prononcée quand les rations sont riches en amidon (ensilage de maïs) ou sur les vaches en acidose. Sur les variations de la composition en $\mathrm{AG}$ du lait, les facteurs déterminants ne sont pas les mêmes selon les AG étudiés. On trouve principalement le type de supplément utilisé (graines crues, graines extrudées, huile), la dose apportée, la teneur en concentrés amylacés de la ration ou le fourrage de la ration de base. Concernant l'effet dose, la majorité des données rapportent un effet linéaire de la dose, la réponse étant globalement proportionnelle à la quantité de lin ajoutée. Les AGS, courts et moyens (C4 à $\mathrm{C} 14)$ répondent par une diminution proportionnelle à la dose de lipides ajoutés, qui est plus prononcée avec des huiles et sur des rations d'ensilage de maïs. Les C18:1-trans augmentent principalement avec les huiles et les graines extrudées, et proportionnellement à la dose de lipides ajoutés. Le C18:3 n-3 augmente en moyenne moins avec l'huile qu'avec les graines, crues ou extrudées, et n'est pas proportionnel à la quantité de lipides apportée dans la ration. Il reste une variabilité inexpliquée pour le moment dans les teneurs atteintes, en moyenne de 0,7 à $0,9 \%$ des $A G$ (entre 0,4 et $1,7 \%)$. Les données disponibles ne montrent pas d'effet important du stade de lactation sur la réponse des vaches à la supplémentation en lin.

Une des principales raisons de l'apport de lin dans la ration des vaches est l'augmentation des teneurs en AG polyinsaturés (principalement le C18:3 n-3, acide linolénique) et la diminution des teneurs en AG saturés. Les niveaux

Figure 2. Production laitière, taux butyreux, pourcentages d'AG saturés et de C18:3, et rapport 18:2/18:3 du lait de vaches Tarentaises et Montbéliardes, en fonction de la ration de base (adapté de Ferlay et al 2006).
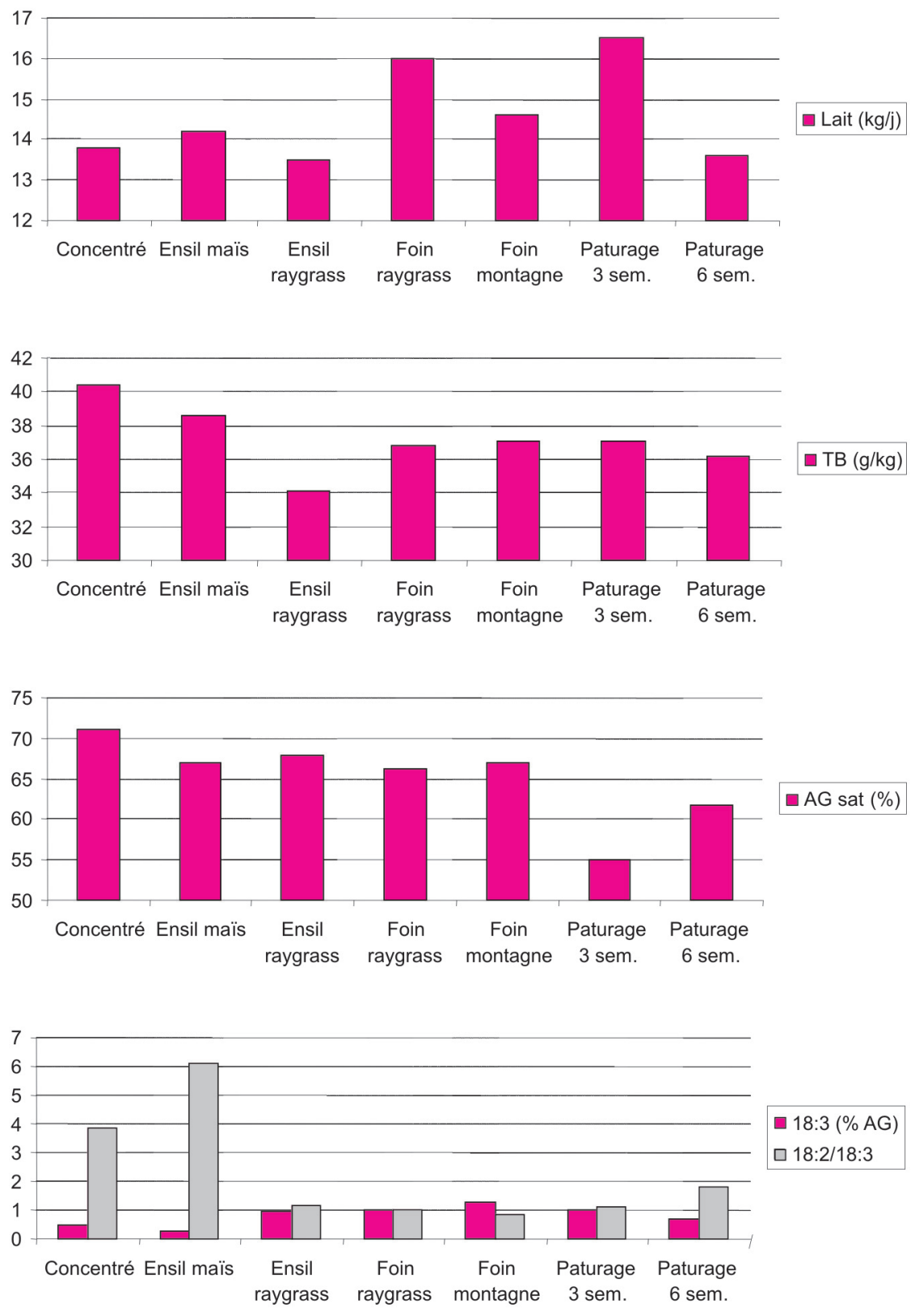
initiaux de ces teneurs, selon les rations de fourrages conservés, sont rappelés dans la figure 2. Si l'on compare les effets du lin à l'herbe, autre source majeure d'acide linolénique, on constate que les teneurs en $\mathrm{C} 18: 3$ sont similaires (Ferlay et al 2006, Farruggia et al 2008). En revanche, la supplémentation en lin induit des concentrations plus fortes en C18:1-trans, en particulier avec les graines extrudées et l'huile (entre 1,5 et 5\% de C18:1-trans pour des régimes à base $\mathrm{d}$ 'herbe, contre en moyenne près de $10 \%$ pour des régimes supplémentés avec 400 à $500 \mathrm{~g} / \mathrm{j}$ de lipides sous forme de graines extrudées ou d'huile de lin), comme déjà relevé par Ferlay et al (2008).

Les effets observés suite à l'introduction de lin dans la ration sont donc en partie semblables à ceux rencontrés lors de l'introduction partielle ou totale d'herbe pâturée dans le régime des vaches laitières, à l'exception d'une teneur en AG trans supérieure. L'utilisation de luzerne dans la ration ou de concentré protéique de luzerne en remplacement de tourteau de soja aboutit à des effets analogues, en particulier d'enrichissement en acide linolénique (Peyraud et al 2008).

Le lin dans l'alimentation des vaches ou des brebis laitières modifie la structure physique des produits laitiers sans changement dans leurs qualités organoleptiques. Il semblerait que dans le cadre d'un traitement UHT, ces laits soient plus sensibles à l'oxydation.
La diminution de la production de méthane par la supplémentation en lin dans les rations des vaches paraît bien établie, mais des essais complémentaires sont nécessaires pour mieux quantifier les effets sur leurs performances de reproduction.

\section{Remerciements}

Les auteurs remercient Françoise Labalette (Onidol) de l'information transmise sur la production, les usages et les variétés de lin, ainsi que Catherine Disenhaus (Inra Agrocampus Ouest) pour son avis et la relecture de la partie traitant des effets sur la reproduction.

\section{Références}

AFZ, 2010. io-La Banque de Données de l'Alimentation Animale. www.feedbase.com

Agreste, 2006. Les matières premières de l'alimentation animale en 2006, Chiffres et Données Agroalimentaire, 155, 26p.

Agreste, 2008. http://agreste.maapar.lbn.fr/

Akraim F., Nicot M.C., Juaneda P., Enjalbert F., 2007. Conjugated linolenic acid (CLnA), conjugated linoleic acid (CLA) and other biohydrogenation intermediates in plasma and milk fat of cows fed raw or extruded linseed. Animal, 1, 835-843.

Ambrose D.J., Kastelic J.P., Corbett R., Pitney P.A., Pett H.V, Small J.A., Zalkovic P., 2006. Lower pregnancy losses in lactating dairy cows fed a diet enriched in $\alpha$-linolenic acid. J. Dairy Sci., 89, 3066-3074.

Chilliard Y., 1993. Dietary fat and adipose tissue metabolism in ruminants, pigs and rodents: a review. J. Dairy Sci., 76, 3897-3927.

Chilliard, Y., Glasser F., Ferlay A., Bernard L., Rouel J., Doreau M., 2007a. Diet, rumen biohydrogenation, and nutritional quality of cow and goat milk fat. Eur. J. Lipid Sci. Technol., 109, 828-855.

Chilliard Y., Glasser F., Enjalbert F., Ferlay A., Bocquier F., Schmidely P., 2007b. Données récentes sur les effets de l'alimentation sur la composition en acides gras du lait de vache, chèvre et brebis. Renc. Rech. Rum., 14, 321-328.

Chilliard Y., Ferlay A., Mouriot J., Bernard L., Martin C., Doreau M., 2008. Alimentation des ruminants laitiers et AG trans du lait (aspects digestifs et métaboliques). Les acides gras trans d'origine naturelle dans la chaîne alimentaire laitière. Journée thématique du Centre National Interprofessionnel de l'Economie Laitière. 2 décembre, Paris, France, $2 p$.

Chilliard Y., Martin C., Rouel J., Doreau M., 2009. Milk fatty acids in dairy cows fed whole crude linseed, extruded linseed, or linseed oil, and their relationship with methane output. J. Dairy Sci., 92, 5199-5211.

Côrtes C., da Silva-Kazama D.C., Kazama R., Gagnon N., Benchaar C., Santos G.T.D., Zeoula L.M., Petit H.V., 2010. Milk composition, milk fatty acid profile, digestion, and ruminal fermentation in dairy cows fed whole flaxseed and calcium salts of flaxseed oil. J. Dairy Sci., 93, 3146-3157.

Deaville E.R., Givens D.I., Blake J.S., 2004. Dietary supplements of whole linseed and vitamin $E$ to increase levels of $\alpha$-linolenic acid and vitamin $\mathrm{E}$ in bovine milk. Anim. Res., 53, 3-12.

Dewhurst R.J., Shingfield K.J., Lee M.R.F., Scollan N.D., 2006. Increasing the concentrations of beneficial polyunsaturated fatty acids in milk produced by dairy cows in high-forage systems. Anim. Feed Sci. Technol., 131, 168-206.

Farruggia A., Martin B., Baumont R., Prache S., Doreau M., Hoste H., Durand D., 2008. Quels intérêts de la diversité floristique des prairies permanentes pour les ruminants et les produits animaux ? Inra Prod. Anim., 21, 181200 .

Ferlay A., Martin B., Pradel P., Coulon J.B., Chilliard Y., 2006. Influence of grass-based diets on milk fatty acid composition and milk lipolytic system in Tarentaise and Montbeliarde cow breeds. J. Dairy Sci., 89, 4026-4041.

Ferlay A., Agabriel C., Sibra C., Journal C., Martin B., Chilliard Y., 2008. Tanker milk variability in fatty acids according to farm feeding and husbandry practices in a French semi-mountain area. Dairy Sci. Technol., 88, 193-215.

Ferlay A., Martin B., Lerch S., Gobert M., Pradel P., Chilliard Y., 2010. Effects of supplementation of maize silage diets with extruded linseed, vitamin $\mathrm{E}$ and plant extracts rich in polyphenols, and morning $v$. evening milking on milk fatty acid profiles in Holstein and Montbeliarde cows. Animal, 4, 627-640.

Flachowsky G., Erdmann K., Huther L., Jahreis G., Mockel P., Lebzien P., 2006. Influence of roughage/concentrate ratio and linseed oil on the concentration of trans-fatty acids and conjugated linoleic acid in duodenal chyme and milk fat of late lactating cows. Arch. Anim. Nutr., 60, 501-511.

Flowers G., Ibrahim S.A., AbuGhazaleh A.A., 2008. Milk fatty acid composition of grazing dairy cows when supplemented with linseed oil. J. Dairy Sci., 91, 722-730.
Friggens N.C., Disenhaus C., Petit H.V., 2010. Nutritional sub-fertility in the dairy cow: towards improved reproductive management through a better biological understanding. Animal, 4, 1197-1213.

Glasser F., Ferlay A., Chilliard Y., 2008. Oilseed lipid supplements and fatty acid composition of cow milk: A meta-analysis. J. Dairy Sci., 91, 4687-4703.

Gobert M., Martin B., Ferlay A., Chilliard Y., Graulet B., Pradel Ph., Bauchart D., Durand D., 2009. Plant polyphenols associated to vitamin E efficiently reduce plasma lipoperoxidation in dairy cows given n-3 polyunsaturated fatty acids. J. Dairy Sci., 92. 6095-6104.

Gòmez-Cortés P., Bach A., Luna P., Juárez M., de la Fuente M.A., 2009. Effects of extruded linseed supplementation on n-3 fatty acids and conjugated linoleic acid in milk and cheese from ewes. J. Dairy Sci., 92, 4122-4134.

Gonthier C., Mustafa A.F., Ouellet D.R, Chouinard P.Y., Berthiaume R., Petit H.V., 2005. Feeding micronized and extruded flaxseed to dairy cows: effects on blood parameters and milk fatty acid composition. J. Dairy Sci., 88, 748-756.

Goudédranche H., Fauquant J., .Maubois J.L., 2000. Fractionation of globular milk fat by membrane microfiltration. Lait, 80, 93-98.

Hurtaud C., Faucon F., Couvreur S., Peyraud J.L., 2010. Linear relationship between increasing amounts of extruded linseed in dairy cow diet and milk fatty acid composition and butter properties. J. Dairy Sci., 93, 1429-1443.

Leroy J.L.M.R., Opsomer G., Van Soom A., Gooovaerts I.G.F., Bols P.E.J., 2008. Reduced fertility in high yielding dairy cows: are the oocyte and embryo in danger? Part 1: the importance of NEB and altered corpus luteum function to the reduction of oocyte an embryo quality in high yielding dairy cows. Reprod. Dom. Anim., 43, 612-622.

Lessard M., Gagnon N., Petit H.V., 2003. Immune response of postpartum dairy cows fed flaxseed. J. Dairy Sci., 86, 2647-2657.

Loor J.J., Ferlay A., Ollier A., Doreau M., Chilliard Y., 2005. Relationship among trans 
and conjugated fatty acids and bovine milk fat yield due to dietary concentrate and linseed oil. J. Dairy Sci., 88, 726-740

Luna P., Fontecha J., Juárez M., de la Fuente M.A., 2005. Changes in the milk and cheese fat composition of ewes commercial supplements containing linseed with special reference to the CLA content and isomer composition. Lipids, 40, 445-454.

Luna P., Bach A., Juarez M., de la Fuente M.A., 2008. Influence of diets rich in flaw seed and sunflower oil on the fatty acid composition of ewes'milk fat especially on the level on conjugated linoleic acid, n-3 and n-6 fatty acids. Int. Dairy J., 18, 99-107.

Manuel des Corps Gras, 1992. Lavoisier Ed., Tec et Doc, $156 \mathrm{p}$.

Martin B., Lerch S., Ferlay A., Verdier-Metz I., Cornu A., Montel M.C., Pradel P., Chilliard Y., 2009. Extruded linseed and antioxidant supplementation of dairy cow diets: what are the influences on the milk and cheese sensory quality? $9^{\text {th }}$ Int. Meeting Mountain Cheeses, Sainte Eulalie, France, 14-15.

Martin C., Rouel J., Jouany J.P., Doreau M., Chilliard Y., 2008. Methane output and diet digestibility in response to feeding dairy cows with crude linseed, extruded linseed, or linseed oil. J. Anim. Sci., 86, 2642-2650.

Martin C., Ferlay A., Chilliard Y., Doreau M., 2009. Decrease in methane emissions in dairy cows with increase in dietary linseed supply. BSAS Annual Conference, 2009. Proc. Brit. Soc. Anim. Sci., 21.

Martin C., Pomiès D., Ferlay A., Rochette Y., Eugène M., Martin B., M. Doreau, Chilliard Y., 2010. Methane output in dairy cows in response to long-term feeding of grassbased diets supplemented with linseed or rapeseed. $4^{\text {th }}$ Int. Conf. Greenhouse Gases and Animal Agriculture (GGAA), Banff, Canada, abstract.

Michalski M.C., 2004. Milkfat globules: physico-chemical properties as a function of size. Bull. Int. Dairy Fed., 389, 104-107.

Morris D., Diskin M., 2008. Effect of progesterone on embryo survival. Animal, 2, 1112-1119.

Mustafa A.F., Chouinard P.Y., Christensen D.A., 2003. Effects of feeding micronised flaxseed on yield and composition of milk from Holstein cows. J. Sci. Food Agric., 83, 920-926.

Palmquist D.L., Beaulieu A.D., Barbano D.M., 1993. Feed and animal factors influen- cing milk fat composition. J. Dairy Sci., 76 , 1753-1771.

Petit H.V., 2010. Review: Feed intake, milk production and milk composition of dairy cows fed flaxseed. Can. J. Anim. Sci., 90, 115-127.

Petit H.V., Twagiramungu H., 2006. Conception rate and reproductive function of dairy cows fed different fat sources Theriogenology, 66, 1316-1324.

Petit H.V., Benschaar C., 2007. Importance de la nature des graisses alimentaires sur la reproduction des vaches laitières. Renc. Rech. Rum., 14, 329-332.

Petit H.V., Dewhurst, R.J., Scollan N.D. Proulx J.G., Khalid M., Haresign W. Twagiramungu H., Mann G.E., 2002. Milk production and composition, ovarian function, and prostaglandin secretion of dairy cows fed omega-3 fats. J. Dairy Sci., 85, 889-899.

Petit H.V., Côrtes C., Gagnon N., Palin M.F. Tao S., Benchaar C., Lacasse P., 2009. Flax hulls and oil supplementation on the activity of antioxidant enzymes in dairy cows. In: Ruminant physiology. Digestion, metabolism, and effects of nutrition on reproduction and welfare. Chilliard Y., Glasser F., Faulconnier Y., Bocquier F., Veissier I., Doreau M. (Eds) Proc. XIth Int. Symp. Ruminant Physiology September 6-9, Clermont-Ferrand, France, 630-631.

Peyraud J.L., Delaby L., Nozière P., Hurtaud C., 2008. Détermination de la valeur azotée du concentré protéique de luzerne et de ses effet sur la composition des laits. Renc. Rech. Rum. 15,288

Proléa, 2008. De la production à la consommation : France, Europe, Monde. Statistiques des oléagineux et protéagineux, 2007-2008, 2008. Proléa Documentation Ed., 134p.

Sauvant D., Perez J.-M., Tran G., 2004 Tables INRA-AFZ de composition et de valeur nutritive des matières premières destinées aux animaux d'élevage : porcs, volailles, bovins, ovins, caprins, lapins, chevaux, poissons 2 ème Edition revue et corrigée. Inra Ed., Paris, France, 301p

Schmitt B., Ferry C., Daniel N., Weill P., Kerhoas N., Legrand P., 2006. Effet d'un régime riche en acides gras omega 3 et en CLA 9-cis, 11-trans sur l'insulinorésistance et les paramètres du diabète de type 2. OCL, 13, 70-75.

Schori F., Fragnière C., Schaeren W., Stoll W., 2006. Graines de lin et de tournesol dan l'alimentation de la vache laitière. Rev. Suisse Agric., 38, 25-30.
Shingfield K.J., Chilliard Y., Toivonen V., Kairenius P., Givens D.I., 2008. Trans fatty acids and bioactive lipids in ruminant milk. In Bioactive components of milk. Bösze Z. (Ed). Springer, USA. Adv.Exp. Med. Biol., 606, 3-65.

Smet K., De Block J., De Campeneere S., De Brabander D., Herman L., Raes K., Dewettinck K., Coudijzer K., 2009. Oxidative stability of UHT milk as influenced by fatty acid composition and packaging. Int. Dairy J., 19, 372-379.

Smet K., Coudijzer K., Fredrick E., De Campeneere S., De Block J., Wouters J., Raes K., Dewettinck K., 2010. Crystallization behavior of milk fat obtained from linseed-fed cows. J. Dairy Sci., 93, 495-505.

Soita H.W., Meier J.A., Fehr M., Yu P., Christensen D.A., McKinon J.J., Mustafa A.F., 2003. Effects of flaxseed supplementation on milk production, milk fatty acid composition and nutrient utilization by lactating dairy cows. Arch. Anim. Nutr., 57, 107-116.

Stoll W., Sollberger H., Collomb M., Schaeren W., 2003. Graine de colza, de lin et de tournesol dans l'alimentation de la vache laitière. Rev. Suisse Agric., 35, 213-218.

Vanholder T.,. Leroy J.L.M.R, Soom A.V., Opsomer G., Maes D., Coryn M., Kruif A.D., 2005. Effect of non-esterified fatty acids on bovine granulosa cell steroidogenesis and proliferation in vitro. Anim. Reprod. Sci., 87, 33-44.

Vanholder T.,. Leroy J.L.M.R, Van Soom A., Maes D., Coryn M., Fiers T., de Kruif A., Opsomer G., 2006. Effect of non-esterified fatty acids on bovine theca cell steroidogenesis and proliferation in vitro. Anim. Reprod. Sci., 92, 51-63.

Zachut M., Dekel I., Lehrer H., Arieli A., Livshitz, L., Yakoby S, Moallem U., 2010. Effects of dietary fats differing in $n-6: n-3$ ratio fed to high-yieding dairy cows on fatty acid composition of ovarian compartments, follicular status, and oocyte quality. J. Dairy Sci., 93, 529-545.

Zhang R.H., Mustafa, A.F., Zhao X, 2006a. Blood metabolites and fatty acid composition of milk and cheese from ewes fed oilseeds. Can. J. Anim. Sci., 86, 547-556.

Zhang R.H., Mustafa A.F., Zhao X., 2006b. Effects of flaxseed supplementation to lactating ewes on milk composition, cheese yield, and fatty acid composition of milk and cheese. Small Rumin. Res., 63, 233-241.

\section{Résumé}

La supplémentation en lin des rations des vaches laitières est une pratique qui se développe, avec pour objectifs l'amélioration de la qualité nutritionnelle du lait et la diminution des rejets de méthane. En effet, la recherche de laits moins riches en Acides Gras (AG) saturés et avec un rapport plus faible entre acide linoléique et acide linolénique, incite à utiliser des aliments riches en acides gras polyinsaturés (et en particulier en acide alpha-linolénique C18:3 n-3) pour corriger des rations insuffisamment riches en cet AGPI. Parmi les aliments des vaches laitières, le lin est un aliment particulièrement riche en C18:3 n-3. La diminution des rejets de gaz à effet de serre (dont le méthane) est également une préoccupation actuelle des filières animales. De nombreux essais de supplémentation en lin, sous différentes formes, ont été publiés ces dernières années, et les données disponibles permettent de tirer des conclusions sur ces effets attendus.

Le présent article fait le point sur les disponibilités en lin et sur les différentes formes d'apport dans les rations. Les effets du lin sur la production laitière, sur la composition du lait et des produits laitiers, la production de méthane et la reproduction sont passés en revue. L'analyse des effets sur le lait s'appuie sur 41 essais zootechniques publiés.

La culture de lin oléagineux est peu importante en France. L'approvisionnement est fait dans des pays européens et au Canada. Les variétés présentent des teneurs variables en acide alpha-linolénique. 
L'introduction de lin dans la ration diminue un peu la quantité de MS ingérée mais ne modifie en général pas la production laitière (volumes et taux). La teneur du lait en AG saturés diminue et le pourcentage en C18:1-trans est augmenté, et ce d'autant plus que l'apport de lipides se fait sous forme non protégée (graines extrudées, huile) et avec des rations riches en amidon (pour les AG trans). La teneur en C18:2 n-6 n'est en moyenne pas modifiée, sauf par l'apport d'huile. La proportion en C18:3 n-3 du lait est multipliée en moyenne par 2 ou 3 pour les formes pratiques les plus efficaces (graines aplaties, farine), et peut atteindre jusqu'à 1,4\% des AG du lait avec ce type de supplémentations. Il n'apparaît pas d'effet dose de lipides apportée pour le C18:2 n-6 et le C18:3 n-3, alors qu'il en existe un pour les C18:1-trans.

Le beurre et les fromages ont la même composition en AG que le lait dont ils proviennent. Les qualités organoleptiques de beurres et fromages ne sont pas modifiées par l'addition de lin dans la ration. Différents effets sont cités dans la bibliographie pour expliquer une augmentation potentielle de la fertilité, qui reste à confirmer. La production ruminale de méthane est diminuée par l'ajout de lin dans la ration.

En conclusion, l'ajout de lin à la ration des vaches laitières a des effets analogues à ceux d'introduction d'herbe dans le régime fourrager, à l'exception d'une teneur en AG trans supérieure.

\section{Abstract \\ Linseed supplementation of dairy cows: Effects on performances, methane emissions, milk composition and dairy products}

Supplementation of the dairy cow diet with linseed products is developing, aiming at increasing milk nutritional quality and decreasing methane output. Feedstuffs rich in polyunsatured fatty acids (FA) (especially linolenic acid) are used to complement diets poor in these FA, to produce a milk lower in saturated FA and with a lower linoleic/linolenic ratio. Linseed is indeed rich in linolenic acid. Greenhouse gas mitigation (including methane) is also a concern for the animal sector. Many studies involving linseed supplementation (with various processing treatments) have been published, and now enable to draw some conclusions about its effects.

This article focuses on the supply of linseed and the various processing treatments. The effects of linseed on milk production, the composition of milk and dairy products, methane production and reproduction are reviewed. The results on milk production and composition are based on 41 published trials.

Linseed cultivation is of little importance in France. Linseed in France is mainly supplied from European countries and Canada. Linseed varieties have different levels of linolenic acid.

Dietary supplementation of cows with linseed slightly decreases dry matter intake, without significant effects on milk production. Milk saturated FA content is decreased and milk C18:1-trans are increased, especially with unprotected supplements (oil, extruded seeds). The C18:2 n-6 content is generally unchanged (except with oils) and starch-rich diets(for trans FA). The proportion of C18:3 n-3 is multiplied by 2 to 3 with the most effective treatments (seeds, flour), and can reach $1.4 \%$ of milk FA, with this type of supplement. The amount of lipid added does not significantly affect the milk content in C18:2 n-6 and C18:3 n-3, while it does for the C18: 1-trans.

Butter and cheeses have the same FA composition as the milk used for their production. The organoleptic properties of butters and cheeses are not affected by linseed addition. Different effects are reported in the literature to explain a potential increase in fertility, which remains to be confirmed. Dietary linseed supplementation decreases the production of methane. In conclusion, dietary linseed supplementation of dairy cows has the same effects as those with the addition of grass in the diet, except for a higher content of trans FA.

BRUNSCHWIG P., HURTAUD C., CHILLIARD Y., GLASSER F., 2010. L'apport de lin dans la ration des vaches laitières : Effets sur la production, la composition du lait et des produits laitiers, les émissions de méthane et les performances de reproduction. Inra Prod. Anim., 23, 307-318. 
